\title{
Tratamiento paliativo ambulatorio de disnea con catéter pleural tunelizado en derrame pleural maligno. Estudio descriptivo
}

\author{
Francisco M. Páez-Codeso, $\bowtie$ Antonio Dorado-Galindo, David M. Jiménez-Fernández, \\ Enrique Bermejo-Casero, Carlos Ruarte-Naranjo, Gracia Eugenia González-Angulo, \\ Patricia Gutiérrez-Castaño
}

Hospital Regional Universitario. Málaga. España.

Trabajo recibido: 16-VI-2017; aceptado: 27-IX-2017

\begin{abstract}
RESUMEN. Objetivo: Describir la evolución clínica, hasta su fallecimiento, de un grupo de pacientes con derrame pleural maligno (DPM), a los que se ha colocado un drenaje pleural tunelizado (DPT) para el alivio ambulatorio de la disnea. Método: Incluimos a pacientes hospitalizados en el Hospital Regional de Málaga desde enero hasta diciembre 2016 con DPM, cuyo motivo de ingreso era la disnea por el derrame pleural y fue tratado con colocación de DPT. Describimos la técnica de inserción y origen de tumor primario, histología, características del derrame pleural, complicaciones, calidad de vida y supervivencia. Resultados: Inscribimos al estudio a 21 pacientes; 10 mujeres y 11 hombres, con una media de 70 años (44-94).Tipos tumorales: carcinoma broncogénico, 8 casos (38\%) y no pulmonares el resto. Mejoraron de la disnea, medida por la escala mMRC (Modified Medical Research Council), 15 pacientes (71.4\%) y no hubo reingresos por causa del derrame pleural. Supervivencia media de 47 días (3-174). Tasa de complicaciones 3 casos (14\%). El procedimiento de drenaje en domicilio fue rápidamente aprendido por la familia y no supuso sobrecarga de trabajo al cuidador. Conclusiones: el DPT evita ingresos hospitalarios y síntomas causados por DPM. Mejora la disnea en la mayoría de casos y la calidad de vida. El procedimiento es eficaz, seguro con mínimas complicaciones menores.
\end{abstract}

Palabras clave: Derrame pleural maligno, catéter pleural tunelizado, paciente ambulatorio.

ABSTRACT. Objective: Describe the results of a consecutive group of patients treated with tunnelized indwelling pleural catheter (TIPC) (Pleur $X^{\mathrm{TM}}$ ) for dyspnea caused by malignant pleural effusion (MPE) until their death. Method: We included patients admitted for dyspnea caused by malignant pleural effusion from January to December 2016. We describe the insertion technique and analyse the patients' origin, type of tumour, characteristics of the pleural effusion, complications, the quality of life and survival once the TIPC is inserted. Results: Accrued 21 patients, 11 men and 10 women, average age of 70 years (44-94). Primary histology: bronchogenic carcinoma 8 patients (38\%), the rest being extrapulmonary carcinoma. Dyspnea improved in 15 patients (71\%), by the $\mathrm{mMRC}$ scale. There were no re-admisions for dyspnea and/pleural effusions. Survived for an averge of 47 days (3-174). Complications: $3 / 21$ cases (14\%). The family members quickly learned the home drainage procedure, which did not overwork the caregiver. Conclusions: TIPC prevented readmission due to symptomatic pleural effusion. Symptoms of dyspnea and quality of life improved in majority of patients. The procedure has proven safe, effective with a few minor complications.

Key words: Malignant pleural effusion, catheter tunnelled, management outpatient.

\section{INTRODUCCIÓN}

El control eficiente y duradero de los síntomas asociados con el derrame pleural maligno (DPM) es de gran importancia para los pacientes en etapas terminales o avanzadas de su enfermedad, cuando la calidad de vida se convierte en la meta de su tratamiento. Existen varias formas de tratamientos paliativos para manejarlos. Generalmente el primero y más sencillo es la toracocentesis, que se debe reservar para pacientes muy debilitados con una esperanza de vida menor a un mes, en los que la debilidad no se atribuye solo a la compresión mecánica causada por el DPM.1,2

La recurrencia al mes del DPM después de una toracocentesis es cercana al $100 \%$, por lo tanto, otras estrategias se deben considerar en pacientes con una expectativa de vida mayor a cuatro semanas. Otras opciones más invasivas incluyen colocación de un drenaje pleural con o sin pleurodesis química, catéter tunelizado de permanencia crónica, toracoscopia «mé- 
dica» y talcaje por toracoscopia videoasistida (VATS), y en casos seleccionados pleurectomía. ${ }^{3}$

El cáncer es la primera causa de derrame pleural en los pacientes que tienen indicación clínica de ser sometidos a una toracocentesis, entre el 27 y $37 \% .{ }^{4,5}$ Los tumores primarios más comunes son el de pulmón (37\%) y el de mama (16\%). El DPM, que supone un estadío avanzado de la enfermedad, conlleva un mal pronóstico, constituye una complicación relevante en la toma de decisiones sobre los pacientes y va a tener un gran impacto en la calidad de vida, pues contribuye a un empeoramiento sintomático, sobre todo de la disnea y la necesidad de hospitalizaciones. ${ }^{6}$ Entre los diferentes procedimientos encaminados a mejorar los síntomas tenemos las toracocentesis repetidas, que hacen necesario la asistencia del paciente al hospital, la pleurodesis con talco, para lo que hay que hospitalizarlo, y una relativamente nueva opción ${ }^{7}$ como es un drenaje permanente, que permite el autocontrol de la disnea en el propio domicilio. Este procedimiento consiste en un catéter de drenaje pleural tunelizado subcutáneo (DPT), que puede colocarse durante el ingreso o en régimen ambulatorio. ${ }^{8}$ Diversos estudios han demostrado la eficacia paliativa de estos catéteres, que se asocian con un menor costo al compararlos con el tratamiento habitual con más reingresos y complicaciones. EI DPT provee una reducción de la estancia hospitalaria y menos disnea. $^{9}$

En nuestra Unidad de Técnicas Bronco-Pleurales tenemos experiencia con este tipo de DPT desde 2015. Hemos querido describir la técnica, evaluar la efectividad en la paliación sintomática y la seguridad del DPT, en un grupo de pacientes consecutivos con DPM recidivante y sintomático.

\section{MATERIAL Y MÉTODOS}

Se ha realizado un estudio retrospectivo observacional descriptivo de seguimiento analizando los pacientes a los que se les colocó un DPT por DPM a lo largo del año 2016. Incluimos pacientes ingresados en distintos servicios tanto de nuestro hospital como de otros y cuyo principal motivo para dicha estancia hospitalaria era la persistencia del DPM con disnea a pequeños esfuerzos. Se comprobó la mejoría sintomática habiéndoseles realizado al menos una toracocentesis evacuadora previa. Leyeron y firmaron un documento de consentimiento, y se instruyó a uno o dos familiares en el manejo del drenaje. Para ello se entregó una hoja de instrucciones en papel y un disco CD con un video facilitado por la casa comercial suministradora (PleurX ${ }^{\circledR}$, Becton Dickinson). No se incluyeron DPM con sospecha de infección, esperanza de vida muy corta, inferior a semanas a juicio de su médico responsable o sin apoyo familiar adecuado. Tampoco se incluyeron derrames pleurales multitabicados en la ecografía torácica.

Recogimos la edad, sexo, sitio primario del cáncer, los datos relativos al derrame pleural (localización, cuantía, aspecto, parámetros bioquímicos y citopatología del líquido pleural, las complicaciones derivadas del drenaje y la calidad de vida en relación con la mejoría de la disnea, medida por la escala modificada de la Medical Research Council (mMRC) (tabla 1). ${ }^{10}$ Por último, hemos querido también analizar algunos aspectos del manejo y atención domiciliaria familiar a estos pacientes.

La inserción del catéter pleural tunelizado se hizo en la sala de técnicas del Servicio de Neumología, siempre por el mismo médico neumólogo. Se siguieron escrupulosamente las normas de esterilidad propias para una cirugía menor. Siempre se administró oxígeno por gafas o puntas nasales y se monitorizaba con pulsioxímetro. El protocolo de la intervención (figura 1) fue el siguiente: previa ecografía torácica para elegir el punto de inserción más idóneo, y con la anestesia local con hidrocloruro de mepivacaína al $2 \%$, punción de la cámara pleural con aguja e inserción con técnica de Seldinger modificada a través de guía de alambre con punta en $\mathrm{J}$ atraumática. A unos $5 \mathrm{~cm}$ de ésta, incisión con hoja de bisturí del número 23 , para disección subcutánea con un tunelizador metálico de punta roma dirigido al alambre guía, se extrae el tunelizador al que va unida la parte fenestrada del catéter pleural. Por el alambre guía se coloca el introductor de $16 \mathrm{Fr}$ desprendible o «pelable» y por éste se introduce a su vez el extremo fenestrado

Tabla 1. Escala modificada de la Medical Research Council. Todos los pacientes del estudio se encontraban en clase funcional grado 4 y hospitalizados antes de ser tratados con el drenaje.

\begin{tabular}{cl}
\hline Grado de disnea & \multicolumn{1}{c}{ Actividad con la que aparece } \\
\hline 0 & $\begin{array}{l}\text { Ausencia de disnea al realizar ejercicio } \\
\text { intenso } \\
\text { Disnea al andar de prisa en llano, o al } \\
\text { andar subiendo una pendiente poco } \\
\text { pronunciada } \\
\text { Incapacidad de mantener el paso de } \\
\text { otras personas de su edad caminando } \\
\text { en llano o tener que parar a descansar al } \\
\text { andar en llano a su propio paso } \\
\text { La disnea hace que tenga que parar a } \\
\text { descansar al andar en llano unos 100 m } \\
\text { o pocos minutos } \\
\text { La disnea le impide salir de casa o } \\
\text { aparece con actividades como vestirse/ } \\
\text { desvestirse o asearse }\end{array}$ \\
4 &
\end{tabular}



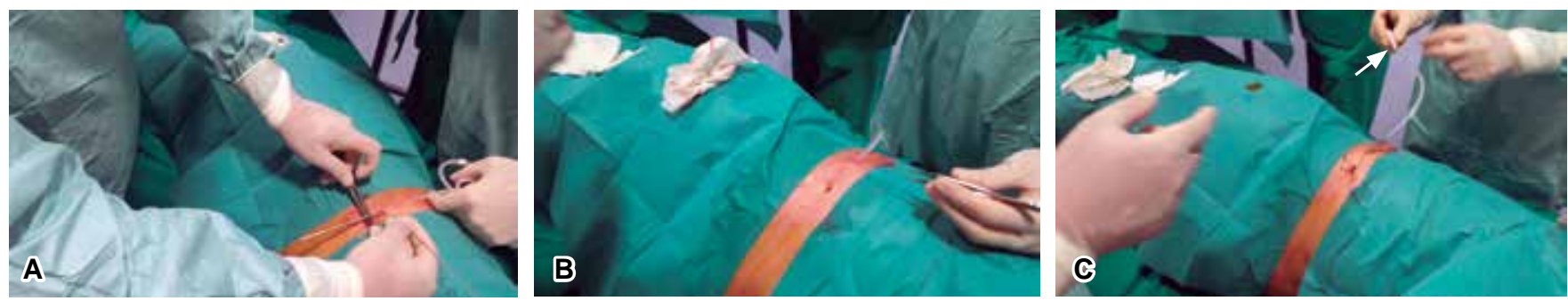

Figura 1. Procedimiento. Momento en el que el catéter es introducido en el tejido subcutáneo con ayuda del tunelizador metálico de punta roma, se aprecia también el alambre guía (A). El catéter recién colocado en su trayecto tunelizado (B). En (C) apreciamos el trayecto de catéter que quedará fuera del tórax, que termina en la válvula unidireccional (flecha).

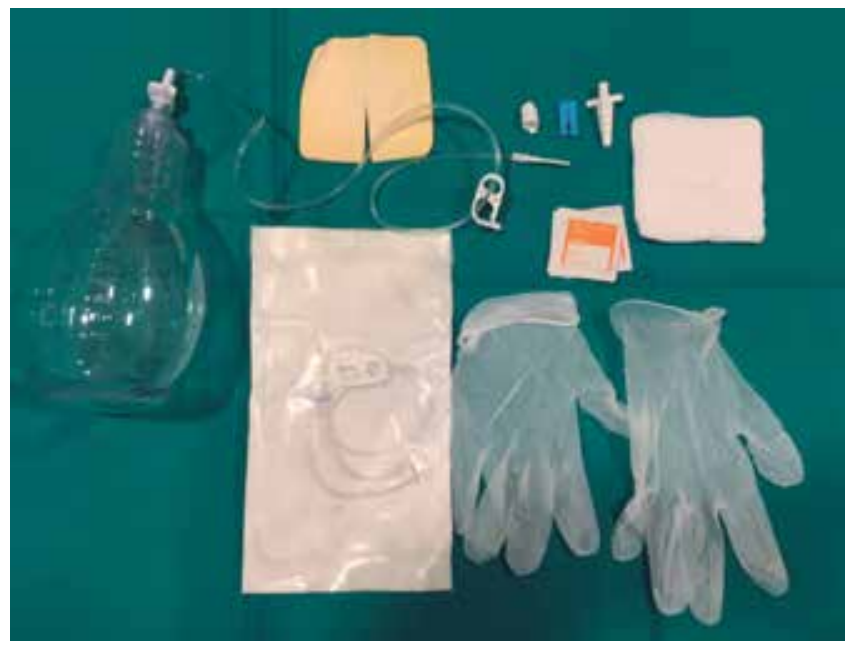

Figura 2. Kit para drenaje domiciliario. Contiene una botella de vacío con tubo de drenaje al final del cual lleva un conector para la válvula unidireccional del catéter, y el material necesario para el cambio de apósitos.

del catéter pleural, asegurándonos que dicho catéter permanece recto en el túnel y sin pliegues ni dobladuras. Por último cierre y fijación con sutura. La intervención lleva unos 30 minutos. El catéter es de silicona, tiene $15.5 \mathrm{~F}$ de calibre y un manguito de poliéster que queda bajo la piel y ayuda a su fijación causada por la fibrosis y cicatrización. El extremo del catéter que queda fuera de la piel termina en una válvula unidireccional que evita el paso de aire o líquido al interior pleural. Esta válvula será la que se conecte al sistema de botellas de vacío para el drenaje (figura 2).

Revisamos ambulatoriamente al paciente entre $7 \mathrm{y}$ 10 días después de la colocación para retirar puntos de sutura y aclarar dudas a la familia, a partir de entonces establecíamos contactos telefónicos y en persona con el familiar que periódicamente pasaba a recoger los recipientes de vacío que iban haciendo falta. En dichos contactos y visitas evaluábamos la mejoría sintomática de la disnea, tomando como referencia la escala de disnea de la mMRC y la independencia para las actividades de la vida cotidiana, así como las posibles complicaciones.

\section{RESULTADOS}

Incluimos a 21 pacientes, 11 hombres y 10 mujeres, con edad media de 70 años (44-94). El origen más frecuente del derrame pleural fue el carcinoma broncogénico, 8 casos (38\%), y tumores extrapulmonares los 13 restantes. De estos últimos, 3 casos de cáncer de colon, 3 de cáncer de mama, 3 casos de carcinoma ovárico y 1 caso de cada uno de los siguientes: hepatocarcinoma, tumor renal, cabeza y cuello y gástrico. Estos datos se sumarizan en la tabla 2 y figura 3 . Todos los pacientes se encontraban en clase funcional 4 de la mMRC e ingresados antes del emplazamiento del drenaje torácico.

\section{Características de los derrames pleurales}

En 13 casos (62\%) el DPM afectaba al hemitórax derecho. En ninguno de nuestros casos hubo DP bilateral significativo. Todos los DPM eran submasivos-masivos. Entendiendo por submasivo aquel que ocupaba entre el 50 al $75 \%$ del hemitórax afecto y masivos los que ocupaban más del $75 \%$.

El aspecto mayoritario resultó serohemático en 16 pacientes (76\%), y amarillento semitransparente el resto. Todos eran exudados por bioquímica pleural, con una media de $4.08 \mathrm{~g} / \mathrm{dL}$ de proteínas y $967.07 \mathrm{UI} / \mathrm{L}$ de lactatodeshidrogenasa (LDH). La citopatología resultó positiva para células neoplásicas en 7 pacientes (33\%).

\section{Evolución de los pacientes}

Los pacientes tras la inserción del DPT recibieron el alta ese día o al siguiente. Como complicación inmediata sólo en un caso evidenciamos una pequeña cámara pleural de neumotórax tras la inserción, resuelta en la 
Tabla 2. Características de los derrames. La escala mMRC (explicada en la tabla 1) se ha expresado como el grado de disnea antes y después del tratamiento con catéter tunelizado. La citopatología del líquido pleural se expresa como positiva (+) o negativa (-). Todos los derrames pleurales tenían una bioquímica correspondiente a exudado.

\begin{tabular}{cccccc}
\hline Caso & Edad & Cuantía, localización, citología & Tumor primario & mMRC & Complicaciones \\
\hline 1 & 71 & Submasivo, derecho (-) & Colon & $4 / 3$ & No \\
2 & 83 & Submasivo, derecho, (-) & Pulmón & $4 / 3$ & No \\
3 & 59 & Masivo, derecho (+) & Colon & $4 / 2$ & Neumotórax resuelto \\
4 & 78 & Submasivo, izquierdo (-) & Colon & $4 / 3$ & No \\
5 & 80 & Masivo, derecho (+) & Pulmón & $4 / 3$ & Salida parcial del catéter \\
6 & 44 & Masivo, izquierdo (-) & Pulmón & $4 / 3$ & No \\
7 & 77 & Masivo, derecho (-) & Hígado & $4 / 4$ & No \\
8 & 72 & Masivo, derecho (+) & Mama & $4 / 2$ & No \\
9 & 61 & Masivo, izquierdo (-) & Rinón & $4 / 3$ & No \\
10 & 83 & Submasivo, izquierdo (-) & Ovario & $4 / 4$ & Salida parcial del catéter \\
11 & 68 & Submasivo, derecho (-) & Mama & $4 / 3$ & No \\
12 & 72 & Masivo, derecho (+) & Cabeza y cuello & $4 / 2$ & Infección espacio pleural \\
13 & 71 & Masivo, izquierdo (+) & Pulmón & $4 / 2$ & No \\
14 & 87 & Masivo, derecho (-) & Pulmón & $4 / 4$ & No \\
15 & 67 & Masivo, derecho (+) & Pulmón & $4 / 2$ & No \\
16 & 69 & Masivo, izquierdo (-) & Gástrico & $4 / 4$ & No \\
17 & 75 & Masivo, derecho (-) & Pulmón & $4 / 2$ & No \\
18 & 78 & Masivo, derecho (-) & Pulmón & $4 / 3$ & No \\
19 & 44 & Masivo, izquierdo (+) & Ovario & $4 / 3$ & No \\
20 & 94 & Masivo, derecho (-) & Mama & $4 / 4$ & $4 / 4$ \\
21 & 49 & Masivo, izquierdo (-) & Ovario & 4 & \\
\hline
\end{tabular}

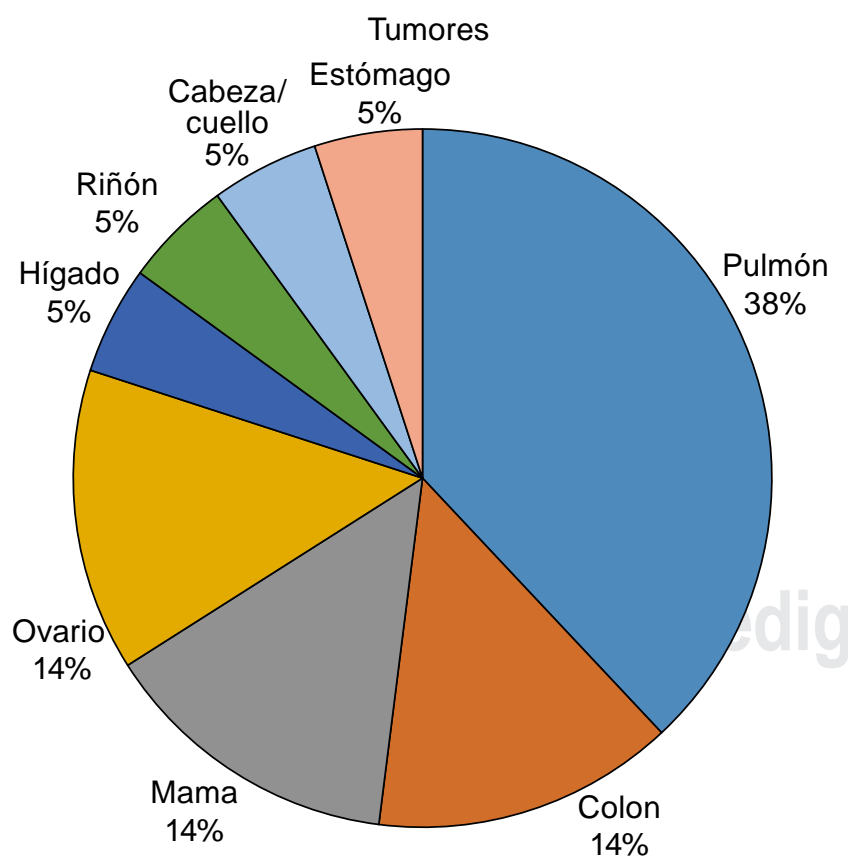

Figura 3. Origen de los tumores que ocasionaron el derrame pleural. Ver texto. visita de revisión una semana después. Y a lo largo de la evolución en dos casos hubo que recolocar el catéter por salida parcial del mismo a través de la incisión. Y un caso de infección del espacio pleural por Pseudomonas aeruginosa multisensible.

En cuanto a la calidad de vida, 15 pacientes (71\%) experimentaron una mejoría de la disnea medida por la escala $\mathrm{mMRC}$, pasando al menos a una clase funcional inferior, permitiendo salidas para paseos cortos fuera del domicilio. Ninguno reingresó por causa del DP (tabla 3). En las entrevistas a los familiares, y por parte del cuidador principal del enfermo, no detectamos miedos, dudas, errores importantes o sobrecarga de trabajo excesiva en el manejo domiciliario del procedimiento de drenaje, manifestando asimismo un aprendizaje rápido del mismo.

Supervivencia, contada a partir del día en que se colocó el DPT, estuvo comprendida entre 3 días y 174, con una media de 47 días. Todos los pacientes habían fallecido en el momento de analizar los datos. No obstante, debemos hacer notar que dada la naturaleza paliativa de este tratamiento, la decisión temporal de colocación del catéter, sería el principal modificador de la supervivencia. 
Tabla 3. Edad, servicio de procedencia, origen del tumor, supervivencia y causa de la muerte del grupo de pacientes.

\begin{tabular}{rccccc}
\hline Número & Edad & Procedencia & Tumor & Supervivencia (días) & Causa de muerte \\
\hline 1 & 71 & Med. Interna & Colon & 13 & Insuf. Respiratoria \\
2 & 83 & C. Paliativos & Pulmón & 14 & Multifactorial \\
3 & 59 & Oncología & Colon & 38 & Progresión enfermedad \\
4 & 78 & C. Paliativos & Colon & 52 & Progresión enfermedad \\
5 & 80 & C. Paliativos & Pulmón & 52 & Insuf. Respiratoria \\
6 & 44 & Oncología & Pulmón & 22 & Insuf. Respiratoria \\
7 & 77 & Digestivo & Hígado & 3 & Encefalopatía hepática \\
8 & 72 & Oncología & Mama & 45 & Progresión enfermedad \\
9 & 61 & Nefrología & Riñón & 31 & Multifactorial \\
10 & 83 & C. Paliativos & Ovario & 46 & Progresión enfermedad \\
11 & 68 & Oncología & Mama & 25 & Progresión enfermedad \\
12 & 72 & Oncología & Cabeza y cuello & 163 & Insuf. Respiratoria \\
13 & 71 & Oncología & Pulmón & 120 & Embolia pulmonar \\
14 & 87 & Neumología & Pulmón & 3 & Insuf. Respiratoria \\
15 & 67 & Oncología & Pulmón & 53 & Progresión enfermedad \\
16 & 69 & Oncología & Estómago & 20 & Progresión enfermedad \\
17 & 75 & Cirugía tórax & Pulmón & 174 & Progresión enfermedad \\
18 & 78 & Med. Interna & Pulmón & 30 & Progresión enfermedad \\
19 & 44 & C. Paliativos & Ovario & 17 & Progresión enfermedad \\
20 & 94 & C. Paliativos & Mama & 60 & Progresión enfermedad \\
21 & 49 & C. Paliativos & Ovario & 15 & Progresión enfermedad \\
\hline
\end{tabular}

\section{DISCUSIÓN}

Toda intervención paliativa destinada a mejorar la calidad de vida en este tipo de pacientes debe tener como objetivo primordial evitar, en la medida de lo posible, el ingreso hospitalario y aliviar la disnea. EI DPT domiciliario ha mejorado la calidad de vida en casi 3 de cada 4 pacientes $(71 \%)$ de la muestra, cumpliendo este objetivo de mejoría sintomática; si bien en la mayoría de casos sólo se logró mejorar la disnea de forma leve-moderada, pasando de un grado funcional 4 a 3 de la mMRC. El DPT evitó en todos los casos el reingreso hospitalario por causa del derrame pleural, lo que ha permitido a muchos de los pacientes su relativa reincorporación a las actividades de la vida cotidiana.

La supervivencia en nuestro grupo de pacientes fue de 47 días, algo por debajo de la comunicada por otros autores que va desde 56 a 144 días, lo cual probablemente quiere decir que la decisión de colocar el DPT se hizo más tardíamente, pero es muy similar -45 días-a la de Cases $\mathrm{E}$ et al. descrita en el único trabajo multicéntrico colaborativo realizado en España. ${ }^{11}$ No obstante, podríamos explicar este dato de supervivencia por la heterogeneidad de los tipos de tumores tan alta en nuestra muestra, con una mayoría de pacientes, 11 de los 21, con tumores extrapulmonares, casi todos muy avanzados con metástasis a distancia, y 6 casos (3 cánceres ováricos, el hepatocarcinoma, el cáncer gástrico y el tumor renal) con carcinomatosis peritoneal ya en el momento del emplazamiento del DPT. Las causas más habituales de fallecimiento registradas en los informes fueron progresión de enfermedad e insuficiencia respiratoria, en 12 y 5 casos, respectivamente.

Hasta la llegada del DPT, ${ }^{12}$ la pleurodesis era la mejor opción de tratamiento del derrame maligno sintomático. Esta técnica pasa por la colocación de un drenaje torácico, hospitalizar al paciente y esperar a que el DP esté totalmente evacuado para en ese momento intentar una sínfisis pleural con talco estéril diluido en suero fisiológico ${ }^{13,14}$ administrado a través del propio drenaje (técnica del «talco slurry»). Sin embargo, son ya varias las publicaciones que abogan por la colocación del DPT como primera opción, pues este método evita la hospitalización, dado que el procedimiento puede ser ambulatorio y ha resultado tan eficaz como la pleurodesis en el control de los síntomas. También sabemos desde hace décadas ${ }^{15}$ que la pleurodesis realizada por cirujanos torácicos a través de una videotoracoscopia, con óptimos resultados, por encima del $90 \%$, hace de ésta una técnica a considerar. Sin embargo, casi ninguno de los pacientes neoplásicos avanzados que tratamos en nuestro centro nos llega en condiciones clínicas de afrontar esta intervención quirúrgica. Por otra parte, la sínfisis pleural con la técnica de «talco slurry» no siempre resulta efectiva. Es en estos casos y en el pulmón atrapado donde el DPT encuentra su mejor e 
indiscutible indicación. Hay que decir también que, a pesar de su eficacia, el talcaje pleural no está exento de posibles complicaciones que aunque muy poco frecuentes, pueden llegar a ser muy graves, como el distrés respiratorio. Así, prestigiosos autores han dado argumentos tanto a favor como en contra de su uso en el derrame maligno. ${ }^{16,17}$

En cuanto a la seguridad del procedimiento, las complicaciones que hemos encontrado no han sido muchas ni importantes. Podemos decir, al igual que en otras recientes publicaciones, ${ }^{18,19}$ que la técnica es segura y con una baja tasa de complicaciones importantes, hecho puesto de manifiesto en series tan largas como la de Tremblay y Michaud. ${ }^{20}$ En el caso de la infección por Pseudomonas aeruginosa optamos por no retirar el drenaje, sino que se trató con antibioterapia oral según antibiograma y drenaje diario (aunque no hubiera disnea) con la intención de mantener el espacio pleural vacío permanentemente. No hubo clínica infecciosa ni síndrome febril achacable a la infección. Aunque en los controles bacteriológicos del líquido seguía cultivándose el microorganismo. El paciente falleció 28 días después del primer cultivo positivo por tromboembolismo pulmonar.

Hemos de poner de manifiesto las limitaciones de nuestro estudio, por una parte es una experiencia inicial con un número pequeño de pacientes, es sólo un estudio descriptivo y no hemos comparado nuestros resultados con el talcaje, además, existen muy pocos ensayos que comparen este particular en la bibliografía. Por todo ello, éste es un tema en el que las conclusiones deben ser precavidas pues queda mucho por decir.

Concluimos que el control domiciliario de la disnea, ocasionada por el derrame pleural maligno sintomático y recidivante, con catéteres tunelizados, realizado de forma ambulatoria por la propia familia del paciente y asistidos por un equipo médico especializado, es una opción razonable de tratamiento, segura y eficaz en la paliación de los síntomas, reduce y evita ingresos hospitalarios por esta causa.

Por ello creemos interesante que las características de esta modalidad de tratamiento sean más conocidas por los profesionales de Atención Primaria, Unidades de Medicina Paliativa, Geriatría y Oncología.

\section{REFERENCIAS}

1. Beyea A, Winzelberg G, Stafford RE. To drain o not to drain: an evidence-based approach to palliative procedures for the management of malignant pleural effusions. J Pain Symptom Manage 2012;44(2):301-306. doi: 10.1016/j.jpainsymman.2012.05.002.
2. Musani Al. Treatment options for malignant pleural effusion. Curr Opin Pulm Med 2009;15(4):380-387 doi: 10.1097/MCP.0b013e32832c6a8a.

3. Murthy SC, Rice TW. Surgical management of malignant pleural effusions. Thorac Surg Clin 2013;23(1):43-49. vi. doi: 10.1016/j.thorsurg.2012.10.001.

4. Villena V, López-Encuentra A, Echave-Sustaeta J, Álvarez Martínez C, Martín Escribano P. Estudio prospectivo de 1000 pacientes consecutivos con derrame pleural. Etiología del derrame y características de los pacientes. Arch Bronconeumol 2002;38(1):21-26.

5. Porcel JM, Esquerda A, Vives M, Bielsa S. Etiología del derrame pleural: análisis de más de 3.000 toracocentesis consecutivas. Arch Bronconeumol 2014;50(5):161-165. doi: 10.1016/j.arbres.2013.11.007.

6. Cano HM, Priede DL, Carrascosa PM. Tratamiento ambulatorio del derrame pleural maligno: aportación de un caso. Med Paliat 2012;19(1):3-9.

7. Seijó L, Capo A, Alcaide AB, Lacunza MM, Armendáriz AC, Zulueta JJ. Manejo ambulatorio del derrame pleural maligno mediante colocación de un catéter pleural tunelizado. Experiencia preliminar. Arch Bronconeumol 2006;42(12):660-662.

8. Musani Al, Haas AR, Seijo L, Wilby M, Sterman DH. Outpatient management of malignant pleural effusions with small-bore, tunneled pleural catheters. Respiration 2004;71(6):559-566.

9. Putnam JB Jr, Light RW, Rodriguez RM, et al. A randomized comparison of indwelling pleural catheters and doxycycline pleurodesis in the management of malignant pleural effusions. Cancer 1999;86(10):19921999.

10. Brooks SM. Task group on surveillance for respiratory hazards in the occupational setting. Surveillance for respiratory hazards. American Thoracic Society News 1982;8:12-16.

11. Cases E, Seijo L, Disdier C, et al. Uso del drenaje pleural permanente en el manejo ambulatorio del derrame pleural maligno recidivante. Arch Bronconeumol 2009;45(12):591-596. doi: 10.1016/j.arbres.2009.09.009.

12. Zeldin DC, Rodríguez RM. Management of refractory malignant pleural effusions with chronic indwelling pleural catheter. Chest 1991;100:87S.

13. Marín PJF, Moreno VMA, Soriano MM, et al. Suspensión de talco para efusión pleural maligna. Farmacia Hosp (Madrid) 2001;25(4):224-228.

14. Shaw P, Agarwal R. Pleurodesis for malignant pleural effusions. Cochrane Database Syst Rev 2004;(1):CD002916.

15. Rivas de Andrés JJ. Pleurodesis: indicaciones, técnicas y resultados. Arch Bronconeumol 1984;6:56-62.

16. Light RW. Talc should not be used for pleurodesis. Am J Respir Crit Care Med 2000;162(6):2024-2026.

17. Sahn SA. Talc should be used for pleurodesis. Am J Respir Crit Care Med 2000;162(6):2023-2024.

18. Meier M, Mortensen MR, Larsen LU. Implantation of permanent pleural catheter for palliation of malignant pleural effusion. Cancer Manag Res 2016;8:129-133. 
19. Kaifi JT, Toth JW, Gusani NJ, et al. Multidisciplinary management of malignant pleural effusion. J Surg Oncol 2012;105(7):731-738. doi: 10.1002/jso.22100.

20. Tremblay A, Michaud G. Single-center experience with 250 tunnelled pleural catheter. insertions for malignant pleural effusion. Chest 2006;129(2):362-368. $\triangle$ Correspondencia:

Dr. Francisco M Páez-Codeso

C/ Manzanilla Núm. 13. 29190-Málaga, España.

Correo electrónico: broncoscopia.hch.sspa@juntadeandalucia.es paezco64@gmail.com

Los autores declaran no tener conflicto de intereses. 